\title{
Optimal Unit Commitment with Smart Metering Sensors on Short-term Ramp Rate for Independent Power Grid
}

\author{
Shiue-Der Lu and Meng-Hui Wang ${ }^{*}$ \\ Department of Electrical Engineering, National Chin-Yi University of Technology, \\ No. 35, Lane 215, Sec. 1, Chung-Shan Rd., Taiping District, Taichung City 411, Taiwan
}

(Received October 13, 2017; accepted December 20, 2017)

Keywords: thermoelectric and hydroelectric unit, smart power grid, wind and solar energy, smart power metering sensor, improved priority method, unit commitment, filtering-constraint implicit enumeration algorithm, ramp rate

In this study, we considered the flexible dispatch of thermoelectric and hydroelectric units in a smart power grid that includes a considerable amount of wind and solar energy. The parameters required for the generators were analyzed using real-world information provided by power companies through smart power metering sensors. We designed and tested an improved priority method and a filtering-constraint implicit enumeration algorithm to solve a large-scale unit commitment problem by means of nonlinear mixed integer programming. The algorithm, which was developed using MATLAB, avoided falling into local or infeasible solutions. A simulation proved that the priority method and algorithm successfully controlled ramp rates and optimized electrical power delivered through the grid, the system was secure, and the economic costs were minimal.

\section{Introduction}

Current trends suggest that renewable energy will dominate future development trends, and that wind and solar energy will be the focus of future development. However, under changing weather conditions, wind and solar power grids deliver unstable quantities of power. ${ }^{(1)} \mathrm{A}$ conventional unit commitment plan specifies the operating time and the generating capacity for each unit within a deployment that may be as short as one day or as long as three months. A unit commitment plan seeks to minimize the operating cost while conforming to the operating limits of each unit and meeting the load requirements, such as the upper and lower limits of the power generation units, the unit ramp rate, and the minimum start-stop time.

Because renewably generated power can fluctuate wildly within a few minutes because of weather influences (such as solar eclipses, strong winds, or a lack of wind), contingency plans, including prepared spinning reserves or storage systems, or the termination of renewable energy generation in accordance with predictive analysis, have been introduced. ${ }^{(2)}$ A generous spinning reserve or extensive storage equipment can provide great safety for the power grid, at

*Corresponding author: e-mail: wangmh@ncut.edu.tw http://dx.doi.org/10.18494/SAM.2018.1775 
the price of great economic cost; such costs motivate some energy firms to assign a low priority to renewable energy. Other firms implement renewable energy but do not put it to effective use, leading to losses.

On the basis of real-world data provided by power companies, in this study, we investigated how a smart power grid with extensive use of renewable energy can compensate for electricity shortages within ten minutes-particularly under conditions in which the construction of new substations and transmission lines would be difficult and time-consuming. A filteringconstraint implicit enumeration algorithm was combined with an improved priority method to produce an optimal unit commitment strategy for a green-energy smart grid.

Regarding unit commitment problems, previously, engineers were just expected to provide sufficient electricity to satisfy users' needs; thus, most generator unit dispatching is based on previous experience, and the priority method is generally adopted. ${ }^{(3)}$ The underlying principle is to first arrange the units according to their fuel costs; a rise in load demand raises the priority for the parallel operation of units with low fuel costs. When the load demand declines, the desynchronization and off lining of units with high fuel costs are prioritized, after which optimal scheduling is determined using a combination of exhaustive and priority methods.

The exhaustive method is a type of brute-force algorithm that has been used in unit commitment planning for numerous years. ${ }^{(4,5)}$ In this method, all possible solutions are listed and compared to determine the desired solution. However, including all possible combinations into the calculation is time-consuming and requires substantial computer memory, and this approach tends to fall into the curse of dimensionality without determining the optimal solution, causing commitment results to compromise on solution quality and calculation time.

Other algorithms commonly used in unit commitment include simulated annealing (SA) and particle swarm optimization (PSO). SA is a type of random optimization algorithm that maps a problem to a crystalline substance, then simulates the annealing process of that crystalline substance to discover optimal solutions to the problem. It converges slowly because it is a comprehensive random search method. ${ }^{(6)}$ PSO is a type of parallel algorithm that expresses a group of possible solutions as the positions of a group of particles. As the particles move randomly through a search space, their changing positions correspond to alternate solutions, all of which are evaluated with the objective function. ${ }^{(7)}$

In this study, we propose a combination of an improved priority method and a filteringconstraint implicit enumeration algorithm. To avoid the curse of dimensionality, combinations of generator units are screened on the basis of their characteristics; after this, the optimal scheduling is determined through a filtering-constraint implicit enumeration algorithm. In addition, the unit ramp rates and break points are taken into account, because the scheduling results are affected by differing ramp rates under distinct break points.

\section{Sensor System Structure and Problem Description}

\subsection{Sensor system structure of an independent power grid}

Figure 1 shows the sensor system structure of an independent power grid including power companies' dispatch control centers, wind and solar power plants, thermal power plants, and 


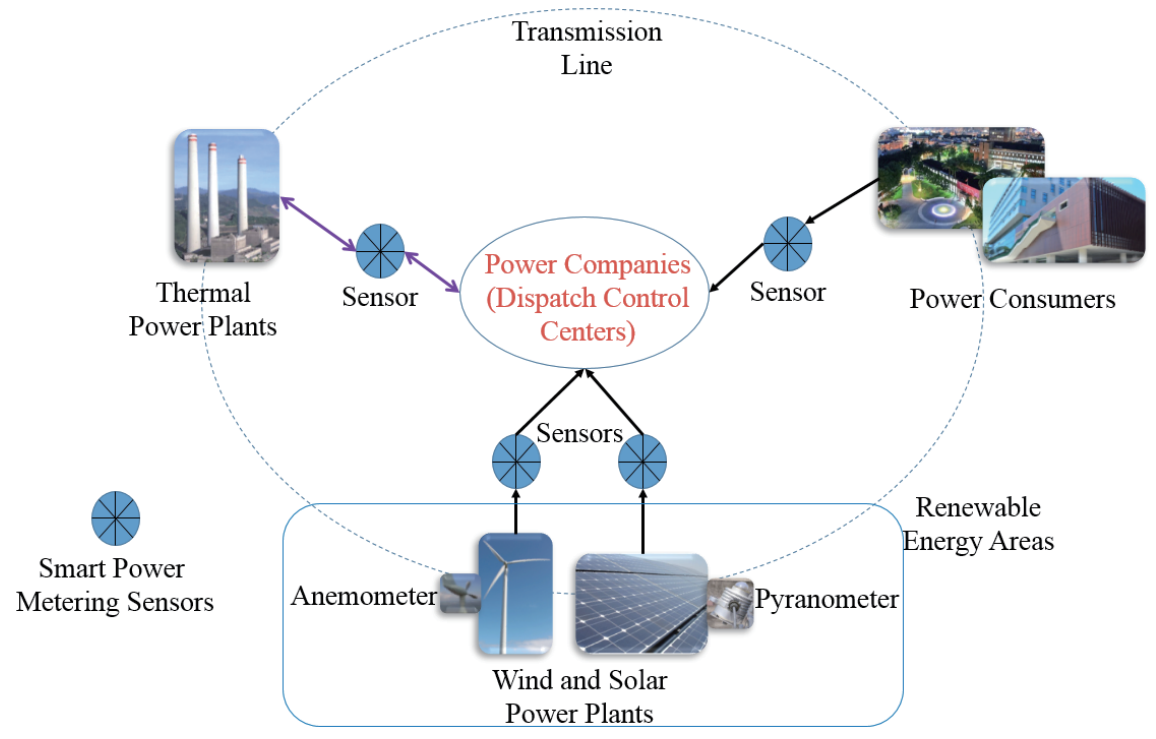

Fig. 1. (Color online) Sensor system structure of an independent power grid.

power consumers. Each power plant and load have their own smart power metering sensors in order to obtain the related electrical signals and transport to the control centers that can calculate the optimal generating power of each thermal unit by the proposed method.

The renewable energy areas include not only pyranometer sensors, wind speed metering sensors, wind direction metering sensors, and so on that are installed to record weather information but also equip smart power metering sensors that are set up to deliver electrical power to the control center.

\subsection{Overview of unit commitment}

Unit commitment determines which generator units must participate in the scheduled operation during a specific period of time. The parameters that require consideration include the number of generators, anticipated load, hot and cold start costs, spinning reserves, and ramp rates. Therefore, the units should not be started and stopped arbitrarily; the load and unit restrictions should be considered to determine when to start and stop the units, while simultaneously maintaining the stability of the system operation.

In this study, we were mainly concerned with the short-term commitment of the units; thus, the start-stop time was not considered. The initial scheduling was conducted using the averaged sum of the maximum and minimum power generation for each unit involved.

The generator units were divided into base load, intermediate load, and peak load.

(a) Base load units are capable of long periods of stable operation, and have low variable cost as well as high rated capacity. Nuclear units and coal-powered units are normally the base loads in power systems.

(b) Peak load units can be quickly activated and stopped, and provide timely compensation for electrical shortages during peak periods in a day. Petroleum-burning units are often used as 
the peak load units with the highest costs in power systems.

(c) For intermediate load units, the power generation cost and operating characteristics fall between the other two unit types. Intermediate load units include liquefied natural gas combined-cycle units; they are also the dispatch units considered in this study.

\subsection{Unit commitment problem}

The dispatch of thermal units focuses on deciding the timing for unit engagement during each interval, as well as the optimal energy generated by each unit, to obtain the system minimal cost (i.e., the objective function). ${ }^{(8)}$

(a) Objective function

$$
\min \sum_{t=1}^{N T} \sum_{i=1}^{N} U_{i}(t) \cdot\left[f_{i}\left(P_{i}(t)\right)+S C_{i}(t)\right]
$$

Here, $N$ is the total number of units, $N T$ is dispatch period (one day or one week), $U_{i}(t)$ is the state of unit $i$ during interval $t, f_{i}\left(P_{i}(t)\right)$ is the fuel cost of unit $i$ during interval $t, P_{i}(t)$ is the generated energy of unit $i$ during interval $t$, and $S C_{i}(t)$ is the start-up cost of unit $i$.

The function of the fuel cost in a thermal unit is expressed with a quadratic equation, as follows:

$$
f_{i}\left(P_{i}\right)=\left(a_{i}+b_{i} P_{i}+c_{i} P_{i}^{2}\right) .
$$

Here, $f_{i}\left(P_{i}\right)$ is fuel cost when the generated energy of unit $i$ is $P_{i}, P_{i}$ is the generated energy of unit $i$, $a_{i}, b_{i}$, and $c_{i}$ are the coefficients of the generating cost of unit $i\left(a_{i}(\$ / \mathrm{h}), b_{i}(\$ / \mathrm{kWh})\right.$, and $\left.c_{i}\left(\$ /(\mathrm{kW})^{2} \mathrm{~h}\right)\right)$.

Besides that, the following system constraints must be considered.

(b) System power balance

$$
\sum_{i=1}^{N} U_{i}(t) \cdot P_{i}=P_{D}
$$

Here, $P_{D}$ is total load demand.

(c) Unit maximal/minimal MW limit

The lifespan of a unit is affected by the thermal limit of a turbine and the minimal flow of water or steam in a boiler. Therefore, to expand the operation lifecycle of a unit, the generated energy of each unit should be limited. When a unit starts up, the minimal energy generated is required but not exceeding the maximal energy. 


$$
P_{i, \min } \leq P_{i} \leq P_{i, \max }
$$

Here, $P_{i, \min }$ is the minimal energy generated by unit $i$, and $P_{i, \max }$ is the maximal energy generated by unit $i$.

(d) Operating reserves

To avoid unexpected load change or unit rejection caused by accidents, sufficient operating reserves should be provided to guarantee reliable power output.

$$
\sum_{i=1}^{N} U_{i}(t) \cdot P_{i, \max } \geq P_{D}+S_{r}(t)
$$

Here, $S_{r}(t)$ is operating reserves during interval $t$.

(e) Start-up cost

The start-up cost is the fuel cost required when a unit generates electrical power in parallel with the power system. A warming process of a unit from shut-off to turn-on is required to increase the temperature and pressure in the boiler before a unit generates electric power. The start-up cost usually corresponds to the unit's up-time. If the unit just shuts off, then the temperature and pressure of the boiler could still remain at a high or medium level. In this case, the start-up cost will be lower, which is called a "hot start-up". In contrast, if the unit has shut off for a long time, then the temperature of the boiler could be at a low level. Under this condition, the start-up cost should be higher, which is called a "cold start-up".

$$
\begin{gathered}
S C_{i}(t)=\left\{\begin{array}{l}
h-\cos t_{i}: M D T_{i} \leq X_{i}^{\text {off }}(t) \leq H_{i}^{\text {off }} \\
c-\cos t_{i}: X_{i}^{\text {off }}(t)>H_{i}^{\text {off }}
\end{array}\right. \\
H_{i}^{\text {off }}=M D T_{i}+c-s-\text { hour }_{i}
\end{gathered}
$$

(f) Unit up/down MW limit

Each unit has a different output range. To fulfill the load changes, their maximum ramp-up and ramp-down ( $R_{\text {iup }}$ and $R_{\text {idown }}$ ) output values in two subsequent hours should be satisfied.

$$
\begin{gathered}
P_{i, t, \max } \geq P_{i, t}+R_{\text {iup }} \\
P_{i, t, \text { min }} \geq P_{i, t}+R_{\text {idown }}
\end{gathered}
$$

The target of this research was to consider the fuel costs and limitation factors to derive a unit commitment plan that minimizes the total cost. 


\section{Proposed Methods for Optimal Unit Commitment}

Figure 2 shows the flow chart for determining the optimal unit commitment. This process involves combining an improved priority method and a filtering-constraint implicit enumeration algorithm.

The proposed method generates solutions as follows. First, the unit parameters for the maximum and minimum power generation and heat consumption are entered. A least-squares method and a curve fitting method are used to obtain the unit fuel cost parameter. An improved priority method is introduced as a rule selection strategy. The screened units are subjected to the filtering-constraint implicit enumeration algorithm to determine whether their costs are the lowest. Finally, the optimal unit schedule is then derived.

The proposed method was run in a MATLAB simulation as follows. The unit parameter data provided by the power companies were analyzed, and the unit fuel cost parameters were obtained by a least-squares method and a curve fitting method. The improved priority method was then adopted for unit screening, after which the filtering-constraint implicit enumeration algorithm was employed to determine the optimal unit scheduling.

\subsection{Least-squares method and curve fitting method}

The fuel cost curve for the thermoelectric units indicates the relationship between cost and output power. The fuel efficiency unit price can be calculated using the fuel price and fuel

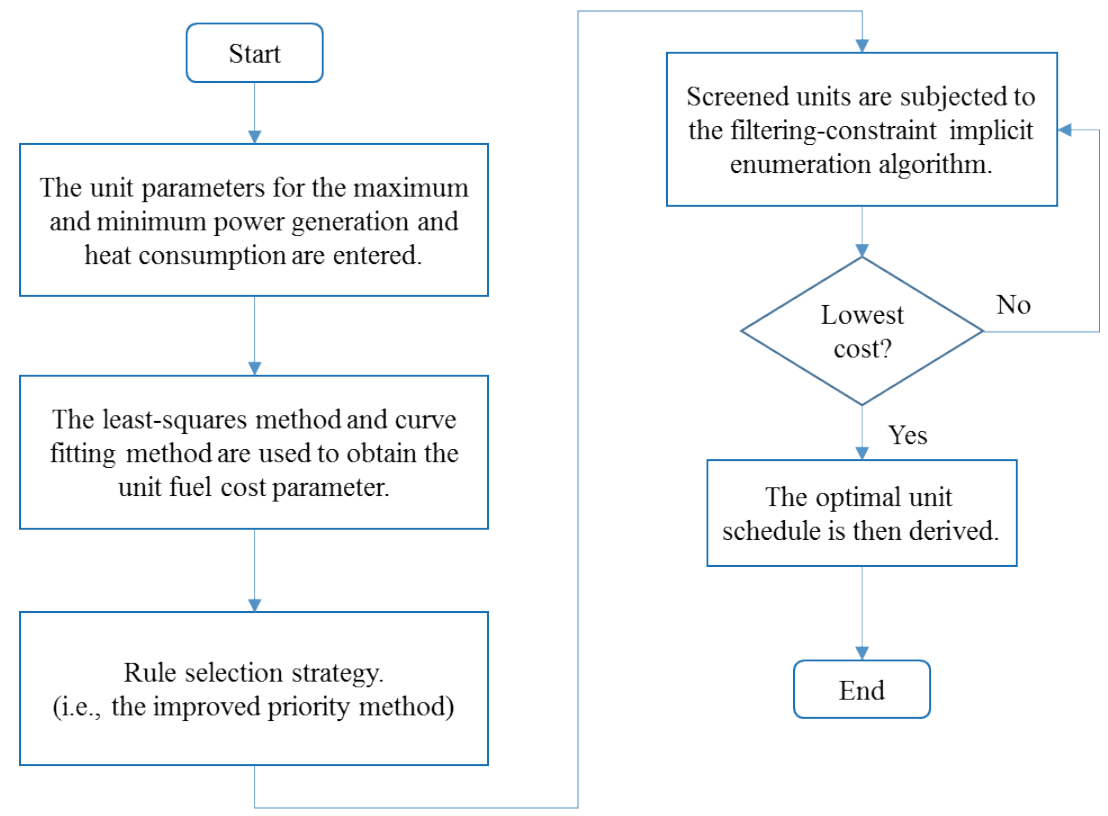

Fig. 2. (Color online) Flow chart of the combination of the improved priority method and filtering-constraint implicit enumeration algorithm. 
efficiency value. The thermal curve value can be obtained by considering the parameters of the generator units, where multiplying the value by the fuel efficiency unit price and power output yields the fuel cost curve.

The unit heat-rate curve indicates the relationship between the heat consumption of fuel and power generation; the vertical axis of the heat-rate curve represents the average heat consumed per MWh, whereas the horizontal axis represents the output power (MW). By using the heat curve of power companies' units as an example, each point on the curve represents the ratio between the thermal input and the generator output. Its definition is shown in Eq. (10). ${ }^{(9)}$

$$
H_{i}=\frac{\alpha^{\prime}}{P_{i}}+\beta^{\prime}+\gamma^{\prime} \times P_{i}
$$

Here, $H_{i}$ is heat rate value (Mkcal/MWh), $P_{i}$ is power generation of the $i$-th unit (MW), and $\alpha^{\prime}$, $\beta^{\prime}$, and $\gamma^{\prime}$ are heat-rate curve parameters.

The fuel cost curve of the units is calculated by multiplying the fuel efficiency unit price by the heat-rate curve parameter and unit power generation, as shown in Eq. (11).

$$
C_{i}=K \times H_{i} \times P_{G i, t}=\alpha_{i}+\beta_{i} \times P_{G i, t}+\gamma_{i} \times P_{G i, t}^{2}
$$

Here, $K$ is fuel unit price $\left(\$ / \mathrm{m}^{3}\right) /$ fuel efficiency $\left(\mathrm{kcal} / \mathrm{m}^{3}\right), P_{G i, t}$ is power generation of the $i$-th unit at time period $t$, and $H_{i}$ is the heat-rate function of the $i$-th unit.

\subsection{Improved rule selection strategy}

The conventional priority method employs a single indicator and uses the average cost of the unit under full load as the priority index; the unit fuel costs are first arranged in descending order. When the load demand increases, the deployment of units with low fuel cost is prioritized, whereas high-cost units are deployed as the peak load units for electricity generation in response to emergencies. This method is simple, easy to implement, and requires a short computation time, although units without a full load or with excessive deviation will yield suboptimal solutions. The conventional and improved priority methods are introduced below.

Because this study focused on methods for achieving short-term unit commitment, which differ from the general priority method, the amount of ramp up should be taken into account during cost calculation using the following equation:

$$
\begin{gathered}
C_{\text {initial }}=\alpha+\beta \times P_{\text {initial }}+\gamma \times P_{\text {initial }}^{2}, \\
C_{\text {up }}=\alpha+\beta \times\left(P_{\text {initial }}+R_{u p} \times t\right)+\gamma \times\left(P_{\text {initial }}+R_{\text {up }} \times t\right)^{2} .
\end{gathered}
$$

Subtract Eq. (12) from Eq. (13) to derive Eq. (14). 


$$
\begin{aligned}
C_{\text {rampup }} & =C_{u p}-C_{\text {initial }} \\
& =\beta \times\left(R_{u p} \times t\right)+\gamma \times\left(P_{\text {initial }}+R_{\text {up }} \times t\right)^{2}-P_{\text {initial }}^{2} \\
& =\beta \times\left(R_{u p} \times t\right)+\gamma \times\left(R_{u p} \times t\right)^{2}+2 \times \gamma \times\left(R_{u p} \times t\right) \times P_{\text {initial }}
\end{aligned}
$$

Here, $C_{\text {initial }}$ is initial power generation cost, $C_{u p}$ is increased power generation cost after ramp up, $C_{\text {rampup }}$ is cost consumed during the ramp-up process, $R_{u p}$ is ramp-up rate (MW/min), and $P_{\text {initial }}$ is initial power.

Divide Eq. (14) by $R_{u p} \times t$ to obtain the average cost for every 1-MW increment, as seen in Eq. (15).

$$
C_{\text {rampup }}=\beta+\gamma \times\left(R_{\text {up }} \times t\right)+\gamma \times P_{\text {initial }}
$$

The parameter $\gamma$ in Eq. (15) affects the ramp-up time of the unit schedule. The selection criteria of the improved priority method are described through the example below.

\subsection{Filtering-constraint implicit enumeration algorithm}

This algorithm involves the addition of a filtering constraint into the limitation factors. To understand its theory and function, consider this example using the method to solve a $0-1$ integer programming problem (in which the variables can only be 0 or 1) in Eq. (16).

$$
\begin{gathered}
\max z=x_{1}-x_{2}+3 x_{3} \\
\text { s.t. }\left\{\begin{array}{l}
2 x_{1}+x_{2}-x_{3} \leq 3 \\
x_{1}+2 x_{2}+2 x_{3} \leq 5 \\
x_{1}+2 x_{2} \leq 4 \\
3 x_{2}+3 x_{3} \leq 7 \\
x_{1}, x_{2}, x_{3}=0 \text { or } 1
\end{array}\right.
\end{gathered}
$$

Solution procedure:

(1) A preliminary feasible solution is obtained; $\left(x_{1}, x_{2}, x_{3}\right)=(1,0,0)$ has met the constraints, with a corresponding objective function value $z=1$.

(2) Because this is a maximization problem, while seeking the optimal solution, any proposal for which $z<1$ is eliminated without checking whether they meet the constraints. Therefore, a new limitation factor known as the filtering constraint is added, as shown in Eq. (17). The original problem is expressed as Eq. (18).

$$
2 x_{1}+x_{2}-x_{3} \geq 1
$$

(3) If the exhaustive method is used for the calculation, then the three variables lead to a total of eight combinations, which are tested in turn to see if they meet the conditions (a)-(e). A proposed combination that does not satisfy (a) does not meet the filtering constraint; thus, tests on (b)-(e) can be omitted to save computation time. 


$$
\begin{gathered}
\max z=x_{1}-x_{2}+3 x_{3} \\
\text { s.t. }\left\{\begin{array}{l}
2 x_{1}+x_{2}-x_{3} \geq 1 \\
2 x_{1}+x_{2}-3 x_{3} \leq 3 \\
x_{1}+2 x_{2}+2 x_{3} \leq 5 \\
x_{1}+2 x_{2} \leq 4 \\
3 x_{2}+3 x_{3} \leq 7 \\
x_{1}, x_{2}, x_{3}=0 \text { (c) } 1
\end{array}\right.
\end{gathered}
$$

(4) If a proposed combination satisfies (a)-(e) and has a corresponding objective value of $z>1$, then the filter constraint is updated until all eight combinations are tested, thereby obtaining the optimal solution $\left(x_{1}, x_{2}, x_{3}\right)=(1,0,1), z=4$.

The objective function is calculated for each proposal in the beginning to test whether it satisfies the filter constraint. The advantage of this method is the reduced amount of calculation, which both accelerates the computation speed and ensures obtaining the optimal solution.

\section{Simulation Results}

\subsection{Simulation results for least-squares method and curve fitting method}

The unit parameters are crucial analytical data for unit scheduling. Unit data were provided by power companies. The unit heat-rate curve and fuel cost curve were calculated by a leastsquares method and a curve-fitting method. Table 1 presents the data for Unit D from a power company, where the measured data from Steps 1-6 are used as fixed points to calculate the heat rate parameters $\alpha, \beta$, and $\gamma$. The calculation is as follows.

Solve the heat-rate function of Unit D.

$$
A X=H \rightarrow\left[\begin{array}{lll}
1 / P_{G 1} & 1 & P_{G 1} \\
1 / P_{G 2} & 1 & P_{G 2} \\
1 / P_{G 3} & 1 & P_{G 3} \\
1 / P_{G 4} & 1 & P_{G 4} \\
1 / P_{G 5} & 1 & P_{G 5} \\
1 / P_{G 6} & 1 & P_{G 6}
\end{array}\right]\left[\begin{array}{c}
\alpha^{\prime} \\
\beta^{\prime} \\
\gamma^{\prime}
\end{array}\right]=\left[\begin{array}{c}
H_{1} \\
H_{2} \\
H_{3} \\
H_{4} \\
H_{5} \\
H_{6}
\end{array}\right]
$$

Table 1

Characteristics of Unit D from a power company.

\begin{tabular}{lcccrrr}
\hline & Step 1 & Step 2 & Step 3 & Step 4 & Step 5 & Step 6 \\
\hline Gross output (MW) & 371 & 456 & 557 & 668 & 743 & 780 \\
Station service load (MW) & 13 & 13 & 14 & 14 & 14 & 14 \\
Net output (MW) & 358 & 432 & 543 & 655 & 729 & 766 \\
Heat consumption (Gcal/h) & 714 & 816 & 968 & 1120 & 1221 & 1272 \\
\hline
\end{tabular}


Here, $P_{G 1}-P_{G 6}$ are the unit net output in Steps $1-6, H_{1}-H_{6}$ are the unit heat consumption/net output in Steps 1-6, and $X$ is calculated by the least-squares method.

$$
\begin{aligned}
A X & =H \\
A^{\mathrm{T}} A X & =A^{\mathrm{T}} H \\
X & =\left(A^{\mathrm{T}} A\right)^{-1} A^{\mathrm{T}} H
\end{aligned}
$$

$X$ is solved.

$$
\begin{aligned}
X & =\left[\begin{array}{ccc}
\alpha^{\prime} & \beta^{\prime} & \gamma^{\prime}
\end{array}\right]^{\mathrm{T}}=\left[\begin{array}{lll}
224.47 & 1.36 & 0
\end{array}\right] \\
& \rightarrow H_{i}=\frac{224.47}{P_{G i, t}}+1.36+0 \mathrm{M} \mathrm{kcal} / \mathrm{MWh}
\end{aligned}
$$

The unit values in Steps 1-6 are then calculated using the polynomial curve-fitting function, as shown by the blue dot in Fig. 3 .

The fuel efficiency unit price (NTD/kcal) is subsequently calculated and multiplied with the heat-rate parameter to obtain the fuel cost parameter. In particular, the fuel efficiency unit price and heat-rate parameter are calculated according to the data provided by power companies, as shown in Table 2; the fuel price is $10.28 \mathrm{NTD} / \mathrm{m}^{3}$, and the fuel efficiency is $9439 \mathrm{kcal} / \mathrm{m}^{3}$.

\subsection{Simulation results for improved priority method}

Real-world data regarding 20 liquefied natural gas combined cycle units were provided by the power companies; the units were screened using the improved priority method to

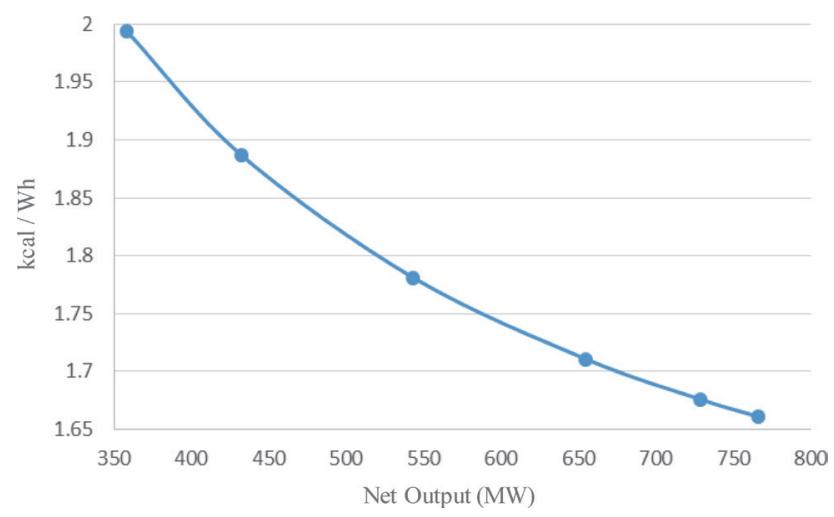

Fig. 3. (Color online) Fitting result of heat-rate curve for Unit D.

Table 2

Fuel efficiency unit price and thermal parameters of Unit D.

\begin{tabular}{lcccc}
\hline & Fuel efficiency & \multicolumn{3}{c}{ Thermal parameters } \\
\cline { 3 - 5 } & unit price (NTD/kcal) & $\alpha^{\prime}$ & $\beta^{\prime}$ & $\gamma^{\prime}$ \\
\hline Unit D & $1.09 \mathrm{E}-3$ & 224.49 & 1.36 & $1.19 \mathrm{E}-7$ \\
\hline
\end{tabular}


compensate for an electricity shortage of $2160 \mathrm{MW}$ within $10 \mathrm{~min}$. The relevant unit parameters are shown in Table 3.

The data in Table 3 were arranged and scheduled on the basis of the improved priority method. When $y=0.047$, the unit numbers under the orange line account for a total of 2082 MW (Fig. 4). The types of unit commitment obtained after screening are as follows:

- Base load units: Units 1, 2, 7, 8, 9, 10, 11, 16, 17

- Peak load units: Units $13,15,18$

- Intermediate load units: Units 3, 4, 5, 6, 12, 14, 19, 20

Here, the dispatch operations for unit scheduling are conducted with: eight intermediate load units. The results are shown in Table 4.

Table 3

Parameters of the 20 thermoelectric units provided by power companies.

\begin{tabular}{|c|c|c|c|c|c|}
\hline & $\alpha$ & $\beta$ & $\gamma$ & Fuel efficiency $\left(\mathrm{kcal} / \mathrm{m}^{3}\right)$ & Fuel price $\left(\mathrm{NTD} / \mathrm{m}^{3}\right)$ \\
\hline Unit 1-2 & -2101 & 44 & $-9.90 \mathrm{E}-3$ & \multirow{14}{*}{9439} & \multirow{2}{*}{ 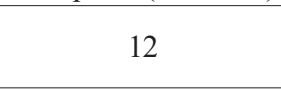 } \\
\hline Unit 3-6 & 16149 & -38 & $6.94 \mathrm{E}-2$ & & \\
\hline Unit 7-8 & 1292 & 20 & $1.97 \mathrm{E}-2$ & & \multirow{12}{*}{11} \\
\hline Unit 9-10 & 15 & 29 & $1.53 \mathrm{E}-4$ & & \\
\hline Unit 11 & -3 & 31 & $-2.50 \mathrm{E}-5$ & & \\
\hline Unit 12 & -5912 & 57 & $-2.00 \mathrm{E}-2$ & & \\
\hline Unit 13 & -10060 & 71 & $-2.70 \mathrm{E}-3$ & & \\
\hline Unit 14 & -328 & 27 & $2.23 \mathrm{E}-2$ & & \\
\hline Unit 15 & -6114 & 50 & $-1.21 \mathrm{E}-3$ & & \\
\hline Unit 16 & -1656 & 38 & $-3.40 \mathrm{E}-4$ & & \\
\hline Unit 17 & -1260 & 30 & $1.85 \mathrm{E}-3$ & & \\
\hline Unit 18 & 2023 & -13 & $1.61 \mathrm{E}-1$ & & \\
\hline Unit 19 & -145 & 42 & $6.47 \mathrm{E}-3$ & & \\
\hline Unit 20 & -301 & 42 & $6.70 \mathrm{E}-3$ & & \\
\hline
\end{tabular}

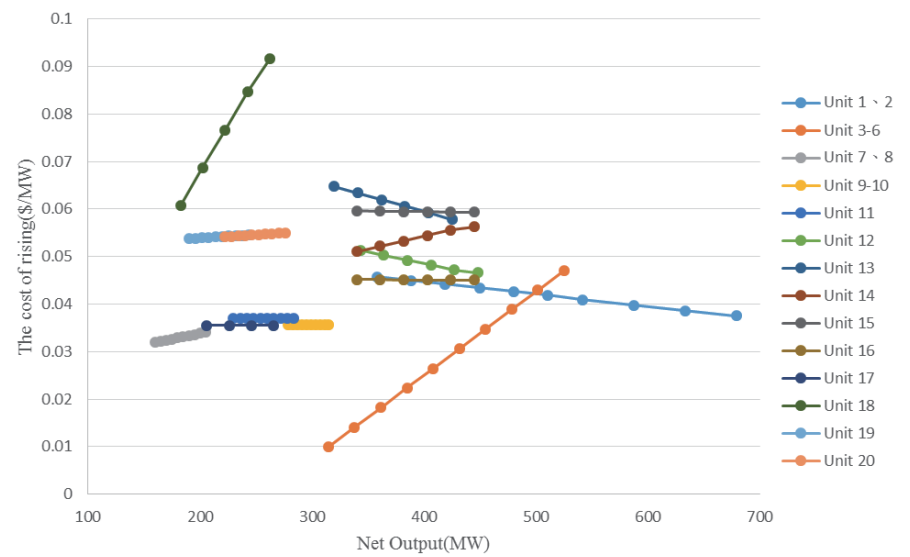

Fig. 4. (Color online) Assessment results of improved priority method for the 20 units. 
Table 4

Scheduling for the 20 units within $10 \mathrm{~min}$.

\begin{tabular}{lcc}
\hline & Ramp up time (min) & \\
\hline Units $1-11$ & 10 & \\
Unit 12 & 5 & Compensate $2160 \mathrm{MW}$ within $10 \mathrm{~min}$. \\
Units $13-15$ & 0 & Total cost: 371450 NTD \\
Unit 16 & 5 & Average computation time: $88-95 \mathrm{~s}$ \\
Unit 17 & 3 & \\
Unit 18 & 0 & \\
Units $19-20$ & 1 & \\
\hline
\end{tabular}

\section{Conclusions}

In the future, a greater proportion of energy is expected to come from renewable sources, and those renewable sources are expected to experience abrupt declines in power. Thus, electrical power shortages are expected, but planners can compensate for gaps in the power grid through appropriate unit scheduling. In this study, we proposed an improved priority method for the prescreening of units to determine the base load units and the units involved in schedule calculation, as well as avoid the curse of dimensionality when all the units participate in the deployment process. Additionally, the filtering-constraint implicit enumeration algorithm was used to obtain the power generation required for each unit while accounting for system security and minimizing economic costs.

\section{References}

1 N. W. Miller: IEEE Power Energy Mag. 13 (2015) 31.

2 W. Xiong, Y. Wang, B. V. Mathiesen, and X. Zhanga: Energy 110 (2016) 55.

3 A. J. Wood and B. F. Wollenberg: Power Generation Operation and Control (Wiley, New York, 1996).

4 K. A. Olsen and B. Indredavik: IEEE Potentials 30 (2011) 18.

5 F. N. Lee: IEEE Trans. Power Syst. 6 (1991) 691.

6 A. Y. Saber, T. Senjyu, T. Miyagi, N. Urasaki, and T. Funabashi: IET Gener. Transm. Distrib. 1 (2007) 234.

7 A. Shukla and S. N. Singh: IET Gener. Transm. Distrib. 10 (2016) 1222.

8 M. T. Kuo and S. D. Lu: JCIE 38 (2015) 547.

9 H. Saadat: Power System Analysis (McGraw-Hill, India, 2002). 